\title{
SUBMODULES OF COMMUTATIVE $C^{*}$-ALGEBRAS
}

\author{
NAZAR MIHEISI \\ Department of Pure Mathematics, University of Leeds, Leeds LS2 9JT, United Kingdom \\ e-mail: naz.miheisi@gmail.com
}

(Received 8 December 2012; accepted 28 May 2013; first published online 13 August 2013)

\begin{abstract}
In this paper we generalise a result of Izuchi and Suárez (K. Izuchi and D. Suárez, Norm-closed invariant subspaces in $L^{\infty}$ and $H^{\infty}$, Glasgow Math. J. 46 (2004), 399-404) on the shift invariant subspaces of $L^{\infty}(\mathbb{T})$ to the non-commutative setting. Considering these subspaces as $C(\mathbb{\mathbb { T }})$-modules contained in $L^{\infty}(\mathbb{\mathbb { T }})$, we show that under some restrictions, a similar description can be given for the $\mathfrak{B}$-submodules of $\mathfrak{A}$, where $\mathfrak{A}$ is a $C^{*}$-algebra and $\mathfrak{B}$ is a commutative $C^{*}$-subalgebra of $\mathfrak{A}$. We use this to give a description of the $\mathbb{M}_{n}(\mathfrak{B})$-submodules of $\mathbb{M}_{n}(\mathfrak{A})$.
\end{abstract}

2010 Mathematics Subject Classification. 46L99, 47A15, 47L30.

1. Introduction. Let $\mathbb{T}$ denote the unit circle $\{z \in \mathbb{C}:|z|=1\}$. A subspace $S$ of $L^{p}(\mathbb{T})$ is said to be shift invariant if for every $f \in S$ we have that the function $z \mapsto z f(z)$ is also in $S$. As is usual, particular importance over the years has been placed on the cases $p=2$ and $p=\infty$. These subspaces, as well as arising naturally in an abundance of purely operator theoretic contexts, have proved important in the study of linear time invariant systems in control theory. A very lucid account of this is given in [7, Chap. 3].

Shift invariant subspaces come in two forms. If $S$ is shift invariant and, in addition, we have that the function $z \mapsto \bar{z} f(z)$ is in $S$ whenever $f \in S$, then $S$ is called doubly invariant or 2-invariant, otherwise it is called simply invariant or 1-invariant. Equivalently, simply invariant subspaces are the shift invariant subspaces $S$ such that $z S \neq S$ and doubly invariant subspaces are those with $z S=S$.

When $p<\infty$, the classification of the closed doubly invariant subspaces is given by Wiener's theorem [7, Theorem 3.1.1]. The classification of the closed simply invariant subspaces is slightly more difficult and is the content of the Beurling-Helson theorem [7, Theorem 3.1.2]. These theorems were then used to derive analogous results for the weak-* closed shift invariant subspaces of $L^{\infty}(\mathbb{T})$. Although this provided a satisfactory classification of these subspaces, much less is know in general about the norm closed shift invariant subspaces of $L^{\infty}(\mathbb{T})$. The most significant progress made so far are the results of Izuchi and Suárez in [5]. In their paper the authors characterised the maximal norm closed simply invariant subspaces of $L^{\infty}(\mathbb{\mathbb { T }})$ and all norm closed doubly invariant subspaces of $L^{\infty}(\mathbb{T})$. In this paper, we will only be considering the latter. For completeness, we will present this result of Izuchi and Suárez [5], but first some definitions will be required.

As usual $\Delta\left(L^{\infty}(\mathbb{T})\right)$ is the spectrum of $L^{\infty}(\mathbb{T})$ and $\hat{f} \in C\left(\Delta\left(L^{\infty}(\mathbb{T})\right)\right)$ is the Gelfand transform of $f \in L^{\infty}(\mathbb{T})$. We regard each bounded Borel measure $\mu$ on $\Delta\left(L^{\infty}(\mathbb{T})\right)$ as a linear functional on $L^{\infty}(\mathbb{T})$ and so we write $\operatorname{ker} \mu$ for the collection of all $f \in L^{\infty}(\mathbb{T})$ 
such that

$$
\int_{\Delta\left(L^{\infty}(\mathbb{T})\right)} \hat{f} \mathrm{~d} \mu=0 .
$$

Let $z$ denote the identity function on $\mathbb{T}$. Then for each $\lambda \in \mathbb{T}$ we define $\mathcal{F}_{\lambda} \subseteq \Delta\left(L^{\infty}(\mathbb{T})\right)$ to be the set of all characters $\varphi \in \Delta\left(L^{\infty}(\mathbb{T})\right)$ such that $\hat{z}(\varphi)=\lambda$. Then $\Delta\left(L^{\infty}(\mathbb{T})\right)=$ $\cup_{\lambda \in \mathbb{T}} \mathcal{F}_{\lambda}$. Define $\Pi$ to be the set of all bounded Borel measures $\mu$ on $\Delta\left(L^{\infty}(\mathbb{T})\right)$ such that $\operatorname{supp} \mu \subseteq \mathcal{F}_{\lambda}$ for some $\lambda \in \mathbb{T}$. We are now in a position to state the theorem.

THEOREM 1. ([5]). A closed subspace $S$ of $L^{\infty}(\mathbb{T})$ is doubly invariant if and only if there is some collection of measures $\Lambda \subseteq \Pi$ such that

$$
S=\bigcap_{\mu \in \Lambda} \operatorname{ker} \mu
$$

We aim to show that this result is, in fact, a special case of more general results describing some of the modules of certain commutative $C^{*}$-algebras. Before proceeding, we will first fix some notation that will be adopted throughout, most of which is standard.

1.1. Notation. Let $\mathcal{H}$ be a Hilbert space. $I$ will denote the identity in $\mathcal{B}(\mathcal{H})$. For a subalgebra $\mathfrak{A} \subseteq \mathcal{B}(\mathcal{H})$, we denote by $(\mathfrak{A})_{1}$ its closed unit ball, $\overline{\mathfrak{A}}^{w}$ its weak closure, $Z(\mathfrak{A})$ its centre and $\mathfrak{A}^{\prime}$ its commutant in $\mathcal{B}(\mathcal{H})$ - that is $\mathfrak{A}^{\prime}=\{T \in \mathcal{B}(\mathcal{H})$ : $T A=A T$ for every $A \in \mathfrak{A}\}$. Given $\psi \in \mathfrak{A}^{*}$ and $A \in \mathfrak{A}$, we will write $A \psi$ to denote the functional $B \mapsto \psi(A B)$ on $\mathfrak{A} . \mathbb{M}_{n}(\mathfrak{A})$ will denote the algebra of all $n \times n$ matrices with entries in $\mathfrak{A}$, although we will simply write $\mathbb{M}_{n}$ rather than $\mathbb{M}_{n}(\mathbb{C})$. $E_{i j}$ denotes the element of $\mathbb{M}_{n}$ with $i, j$ th entry equal to 1 and all other entries 0 .

2. A non-commutative generalisation. It is easily observed that the problem of determining the closed doubly invariant subspaces of $L^{\infty}(\mathbb{T})$ can be thought of as one of determining the closed $C(\mathbb{T})$-modules contained in $L^{\infty}(\mathbb{T})$. It is then natural to ask, if rather than $C(\mathbb{T})$ and $L^{\infty}(\mathbb{T})$ we have two $C^{*}$-algebras $\mathfrak{B}$ and $\mathfrak{A}$ with $\mathfrak{B} \subseteq \mathfrak{A}$, whether we can still give a description of the closed left $\mathfrak{B}$-submodules of $\mathfrak{A}$. We show that under some restrictions on the algebras, a similar description can be given which generalises the result of Izuchi and Suárez [5]. In particular, we will always require $\mathfrak{B}$ to be commutative. In stating and proving the main results, we use many aspects from the non-commutative theory of antisymmetric algebras developed many years ago by Szymanski in $[\mathbf{8}, \mathbf{9}]$. We begin by recalling a basic definition from this theory.

Let $\mathcal{H}$ be a Hilbert space and let $\mathfrak{A} \subseteq \mathcal{B}(\mathcal{H})$ be an operator algebra. A projection $P \in$ $\mathfrak{A}^{\prime}$ is called $\mathfrak{A}$-antisymmetric if for every $A \in \mathfrak{A}$ such that $P A=P A^{*}$, there exists some $r \in \mathbb{R}$ such that $P A=r P$. An $\mathfrak{A}$-antisymmetric projection $P$ is maximal if whenever $Q$ is an $\mathfrak{A}$-antisymmetric projection such that $Q \geq P$, with the standard ordering of projections, we have $Q=P$. It is a straightforward application of Zorn's Lemma to show that every $\mathfrak{A}$-antisymmetric projection is dominated by a maximal one. We denote by $\mathcal{M}(\mathfrak{A})$ the set of all maximal $\mathfrak{A}$-antisymmetric projections. It was shown in [9] that if $\mathfrak{A}$ acts non-degenerately on $\mathcal{H}, \mathcal{M}(\mathfrak{A})$ is contained in the centre of $\overline{\mathfrak{A}}^{w}$ and that the elements of $\mathcal{M}(\mathfrak{A})$ are all orthogonal. For a general operator algebra, indeed even for 
a $C^{*}$-algebra $\mathfrak{A} \subseteq \mathcal{B}(\mathcal{H})$, there need not be any $\mathfrak{A}$-antisymmetric projections (consider, for example, $\left.\mathfrak{A}=\mathcal{B}(\mathcal{H})=\mathbb{M}_{2}\right)$.

We say that $\mathcal{M}(\mathfrak{A})$ is full or that $\mathfrak{A}$ has a full set of antisymmetric projections if $\mathcal{M}(\mathfrak{A})$ is non-empty and

$$
\sum_{P \in \mathcal{M}(\mathfrak{A})} P=I,
$$

where the sum converges in the strong operator topology. It is easily verified that a necessary (but not sufficient) condition for $\mathfrak{A}$ to have a full set of antisymmetric projections is that $\mathfrak{A}$ is commutative.

Throughout the remainder of this section, we fix a Hilbert space $\mathcal{H}$ and two $C^{*}$ -subalgebras $\mathfrak{A}$ and $\mathfrak{B}$ of $\mathcal{B}(\mathcal{H})$ with $\mathfrak{B} \subseteq \mathfrak{A}$. We shall also assume the following:

(1) $\mathfrak{B}$ is commutative with a full set of antisymmetric projections.

(2) $\mathfrak{B}$ (and hence $\mathfrak{A}$ ) acts non-degenerately on $\mathcal{H}$.

We will say that a functional $\psi \in \mathfrak{A}^{*}$ is antisymmetrically supported if for each $P \in$ $\mathcal{M}(\mathfrak{B})$ either $P \psi=\psi$ or $P \psi=0$. We say that a set $\Lambda \subseteq \mathfrak{A}^{*}$ is antisymmetrically supported if every $\psi \in \Lambda$ is antisymmetrically supported.

We can now give a generalisation of Theorem 1 .

THEOREM 2. If every norm continuous linear functional on $\mathfrak{A}$ is ultraweakly continuous, then $M \subseteq \mathfrak{A}$ is a closed left $\mathfrak{B}$-module if and only if there exists an antisymmetrically supported set $\Lambda \subseteq \mathfrak{A}^{*}$ such that

$$
M=\bigcap_{\psi \in \Lambda} \operatorname{ker} \psi .
$$

In order to prove Theorem 2 we will require the following lemma. This is a noncommutative analogue of a result in the commutative theory of uniform algebras, a detailed account of which can be found in [2].

Lemma 3. Assume that every norm continuous linear functional on $\mathfrak{A}$ is ultraweakly continuous and let $M \subseteq \mathfrak{A}$ be a closed left $\mathfrak{B}$-module.

(a) For every $\psi \in \mathfrak{A}^{*}$ we have that if $\psi \in M^{\perp}$ then $\psi \in(P M)^{\perp}$ for each $P \in \mathcal{M}(\mathfrak{B})$.

(b) If $A \in \mathfrak{A}$ and $P A \in P M$ for every $P \in \mathcal{M}(\mathfrak{B})$ then $A \in M$.

Proof. (a) Fix $\psi \in M^{\perp}$ and $P \in \mathcal{M}(\mathfrak{B})$. Since $P \in \overline{\mathfrak{B}}^{w}$, there exists a net $\left(B_{\lambda}\right) \subseteq \mathfrak{B}$ with $B_{\lambda} \rightarrow P$ in the ultraweak topology. As every $\psi \in \mathfrak{A}^{*}$ is ultraweakly continuous, for each $A \in M$ we have $\psi(P A)=\lim _{\lambda} \psi\left(B_{\lambda} A\right)=0$.

(b) Fix $\psi \in M^{\perp}, A \in(\mathfrak{A})_{1}$ and suppose that $P A \in P M$ for every $P \in \mathcal{M}(\mathfrak{B})$. The requirement that $\mathcal{M}(\mathfrak{B})$ is full then ensures that the sum

$$
\sum_{P \in \mathcal{M}(\mathfrak{B})} P A
$$

converges in the strong operator topology (and hence in the weak operator topology) to $A$. Since $\psi$ is ultraweakly continuous, it is weakly continuous on $(\mathfrak{A})_{1}$ 
[6, Proposition 7.4.5], and so

$$
\psi(A)=\sum_{P \in \mathcal{M}(\mathfrak{B})} \psi(P A) .
$$

It then follows from part (a) that $\psi(A)=0$.

We can now proceed to prove Theorem 2.

Proof of Theorem 2. Let $M \subseteq \mathfrak{A}$ be a closed left $\mathfrak{B}$-module. Define $\Lambda \subseteq M^{\perp}$ to be the set of all $\psi \in M^{\perp}$, which are antisymmetrically supported. Firstly, we have the trivial inclusion

$$
M \subseteq \bigcap_{\psi \in \Lambda} \operatorname{ker} \psi
$$

We also see that if $\psi \in(P M)^{\perp}$ for some $P \in \mathcal{M}(\mathfrak{B})$ then $P \psi \in M^{\perp}$. Since $P$ is a projection, we also clearly have that $P \psi$ is antisymmetrically supported and so $P \psi \in \Lambda$. So if $\psi(A)=0$ for every $\psi \in \Lambda$, then $\psi(P A)=0$ for every $\psi \in(P M)^{\perp}$. It then follows from Lemma 3(b) that $A \in M$, and therefore

$$
M=\bigcap_{\psi \in \Lambda} \operatorname{ker} \psi
$$

Conversely, fix $\psi \in \mathfrak{A}^{*}$ and $P \in \mathcal{M}(A)$ such that $P \psi=\psi$. Then for each $B \in \operatorname{ker} \psi$ and $A \in A$,

$$
\psi(A B)=\psi(P A B)=\lambda \psi(B)=0
$$

for some $\lambda \in \mathbb{C}$. So $\operatorname{ker} \psi$ is a left $\mathfrak{B}$-module, and hence an intersection of such things will also be a left $\mathfrak{B}$-module.

EXAMPLE 4. Let $\mathcal{H}$ be a separable Hilbert space with orthonormal basis $\left(e_{n}\right)$. We denote by $\mathcal{K}(\mathcal{H})$ and $\mathcal{D}_{0}(\mathcal{H})$ the algebras of compact operators and compact diagonal operators (with respect to the basis $\left.\left(e_{n}\right)\right)$ respectively. It is straightforward to verify that the $\mathcal{D}_{0}(\mathcal{H})$-antisymmetric projections are rank 1 projections onto subspaces spanned by the basis vectors and that these are in fact maximal so that $\mathcal{M}\left(\mathcal{D}_{0}(\mathcal{H})\right)=\left\{P_{n}: n \in \mathbb{N}\right\}$, where $P_{n}$ is the projection onto the subspace spanned by $e_{n}$. Since every continuous linear functional on $\mathcal{K}(\mathcal{H})$ is induced by a trace class operator, it has an extension to $\mathfrak{B}(\mathcal{H})$, which is ultraweakly continuous $\left(\left[11\right.\right.$, p. 96]). Fix $S \in \mathcal{S}_{1}(\mathcal{H})$, where $\mathcal{S}_{1}(\mathcal{H})$ denotes the trace class operators on $\mathcal{H}$. We will use $\hat{S}$ to denote the functional $T \mapsto$ $\operatorname{tr} S T$. An elementary calculation shows that $P_{n} \hat{S}=\hat{S}$ if and only if $e_{k} \in \operatorname{ker} S$ for every $k \neq n$. Equivalently, $P_{n} \hat{S}=\hat{S}$ if and only if the matrix for $S$ only has non-zero entries in the $n$th column. Then $T \in \operatorname{ker} S$ if and only if the $n, n$th entry of $S T$ is 0 . Consequently, every $\mathcal{D}_{0}(\mathcal{H})$-submodule of $\mathcal{K}(\mathcal{H})$ can be constructed by starting with some collection $\left\{S_{\lambda}\right\} \subseteq \mathcal{S}_{1}(\mathcal{H})$, each member of which will only have non-zero entries in one column, the $n_{\lambda}$ th column say, and then taking all $T \in \mathcal{K}(\mathcal{H})$ such that the $n_{\lambda}, n_{\lambda}$ th entry of $S_{\lambda} T$ vanishes for all $\lambda$.

COROLlary 5. Let $X$ be a closed subalgebra of $\mathfrak{A}$ containing $\mathfrak{B}$. If every norm continuous linear functional on $\mathfrak{A}$ is ultraweakly continuous, then $M$ is a closed left $\mathfrak{B}-$ submodule of $X$ if and only if there exists an antisymmetrically supported set $\Lambda \subseteq \mathfrak{A}^{*}$ 
such that

$$
M=\bigcap_{\psi \in \Lambda} \operatorname{ker} \psi \cap X .
$$

Proof. Fix $P \in \mathcal{M}(\mathfrak{B})$ and suppose that we have $\psi \in \mathfrak{A}^{*}$ with $P \psi=\psi$. For every $B \in \mathfrak{B}$ and $A \in X$ we have that $B A-\lambda A \in \operatorname{ker} \psi$, where $\lambda \in \mathbb{C}$ is such that $P B=\lambda P$. If we also have that $A \in \operatorname{ker} \psi$, then we must have that $B A \in \operatorname{ker} \psi$. Hence, $\operatorname{ker} \psi \cap X$ is a closed left $\mathfrak{B}$-submodule of $X$. Conversely, every closed left $\mathfrak{B}$-submodule of $X$ is trivially a closed left $\mathfrak{B}$-submodule of $\mathfrak{A}$, and so the result follows from Theorem 2 .

EXAMPLE 6. Let $X$ be a closed subalgebra of $L^{\infty}(\mathbb{\mathbb { T }})$ which contains $H^{\infty}(\mathbb{T})$. Such algebras are called Douglas algebras and a detailed account of these is given in [3, Chap. 9]. If we further suppose that $X$ strictly contains $H^{\infty}(\mathbb{T})$, then by Theorems 1.4 and 2.2 of [3] we have that $X$ contains $C(\mathbb{T})$. Corollary 5 then implies that the closed shift invariant subspaces of $X$ are all of the form $S \cap X$, where $S$ is a closed shift invariant subspace of $L^{\infty}(\mathbb{T})$.

We now wish to extend Theorem 2 to give a description of the $\mathbb{M}_{n}(\mathfrak{B})$-submodules of $\mathbb{M}_{n}(\mathfrak{A})$. However, we will first consider $\mathfrak{A}^{n}=\mathfrak{A} \oplus \cdots \oplus \mathfrak{A}$ acting on $\mathcal{H}^{n}$. We can regard $\mathfrak{B}$ as a subalgebra of $\mathfrak{A}^{n}$ by identifying $B \in \mathfrak{B}$ with $(B, \ldots, B) \in \mathfrak{A}^{n}$. It is clear that if every bounded linear functional on $\mathfrak{A}$ is ultraweakly continuous then the same is true for $\mathfrak{A}^{n}$. The definition of antisymmetrically supported elements and subsets of $\mathfrak{A}^{*}$ extends to $\mathfrak{A}^{* n}$ without change, but noting that if $\psi=\left(\psi_{1}, \ldots, \psi_{n}\right) \in \mathfrak{A}^{* n}$ then $P \psi=\left(P \psi_{1}, \ldots, P \psi_{n}\right)$. We are now left with the task of determining the maximal $\mathfrak{B}$-antisymmetric projections in $\mathcal{B}\left(\mathcal{H}^{n}\right)$. It should be noted that despite considering $\mathfrak{B}$ acting on $\mathcal{H}^{n}$ we will reserve $\mathcal{M}(\mathfrak{B})$ exclusively for denoting the maximal $\mathfrak{B}$ antisymmetric projections in $\mathcal{B}(\mathcal{H})$. Let $Q \in \mathcal{B}\left(\mathcal{H}^{n}\right)$ be any maximal $\mathfrak{B}$-antisymmetric projection. Since $Q$ is contained in the weak closure of $\mathfrak{A}^{n}$, we can write $Q=$ $\left(Q_{1}, \ldots, Q_{n}\right)$, where each $Q_{j}$ acts on $\mathcal{H}$. Then it is easy to check that each $Q_{j}$ is a maximal $\mathfrak{B}$-antisymmetric projection in $\mathcal{B}(\mathcal{H})$. Suppose there are indices $j$ and $k$ with $Q_{j} \neq Q_{k}$. Then there is some $B \in \mathfrak{B}$ and distinct complex numbers $\lambda_{j}$ and $\lambda_{k}$ with $B Q_{j}=\lambda_{j} B_{j}$ and $B Q_{k}=\lambda_{k} Q_{k}$. Then $B\left(Q_{j}, Q_{k}\right)=\left(B Q_{j}, B Q_{k}\right)=\left(\lambda_{j} Q_{j}, \lambda_{k} Q_{k}\right) \neq \lambda\left(Q_{j}, Q_{k}\right)$ for any $\lambda \in \mathbb{C}$. It follows that any projection in $\mathcal{B}\left(\mathcal{H}^{n}\right)$ having a subprojection equivalent to $\left(Q_{j}, Q_{k}\right)$ cannot be $\mathfrak{B}$-antisymmetric. So in particular $Q$ is not $\mathfrak{B}$-antisymmetric. We conclude from this that all the $Q_{j}$ are equal and hence $Q=(P, \ldots, P)$ for some $P \in \mathcal{M}(\mathfrak{B})$.

We will now turn our attention to the left $\mathbb{M}_{n}(\mathfrak{B})$-submodules of $\mathbb{M}_{n}(\mathfrak{A})$. We occasionally identify $\mathbb{M}_{n}(\mathfrak{A})$ and $\mathbb{M}_{n}(\mathfrak{B})$ with $\mathfrak{A} \otimes \mathbb{M}_{n}$ and $\mathfrak{B} \otimes \mathbb{M}_{n}$, respectively, when it is convenient to do so. Before continuing, let us agree on a useful convention. We will regard elements of $\mathfrak{A}^{* n}$ as column vectors and if $A=\left(A_{i j}\right) \in \mathbb{M}_{n}(\mathfrak{A}), \lambda=\left(\lambda_{i j}\right) \in \mathbb{M}_{n}$ and $\psi \in \mathfrak{A}^{* n}$ then the 'products' $A \psi$ and $\lambda A$ are the usual ones; however, in this instance we interpret terms, such as $A_{i j} \psi_{k}$, to mean $\psi_{k}\left(A_{i j}\right)$. With this understood, we define for each $\psi \in \mathfrak{A}^{* n}$ a linear map $R_{\psi}: \mathbb{M}_{n}(\mathfrak{A}) \rightarrow \mathbb{C}^{n}$ by setting $R_{\psi} A=A \psi$ for every $A \in \mathbb{M}_{n}(\mathfrak{A})$.

THEOREM 7. If every norm continuous linear functional on $\mathfrak{A}$ is ultraweakly continuous then $M \subseteq \mathbb{M}_{n}(\mathfrak{A})$ is a closed left $\mathbb{M}_{n}(\mathfrak{B})$-module if and only if there is an antisymmetrically supported set $\Lambda \subseteq \mathfrak{A}^{* n}$ such that

$$
M=\bigcap_{\psi \in \Lambda} \operatorname{ker} R_{\psi} .
$$


Proof. Assume that $M \subseteq \mathbb{M}_{n}(\mathfrak{A})$ is a closed left $\mathbb{M}_{n}(\mathfrak{B})$-module. For $i=1, \ldots, n$, let $M_{i}$ be the set of all $\left(A_{i 1}, \ldots, A_{i n}\right) \in \mathfrak{A}^{n}$ such that $A=\left(A_{i j}\right) \in M$. It is clear that each $M_{i}$ is a closed left $\mathfrak{B}$-module and so there exist antisymmetrically supported sets $\Lambda_{1}, \ldots, \Lambda_{n} \subseteq \mathfrak{A}^{* n}$ such that

$$
M_{i}=\bigcap_{\psi \in \Lambda_{i}} \operatorname{ker} \psi .
$$

We claim that all the $\Lambda_{i}$ are equal. Fix $i$ and $j$ with $1 \leq i, j \leq n$. Since $M$ is a left $\mathbb{M}_{n}(\mathfrak{B})$ module, we have in particular that for every $B \in \mathfrak{B}$ and every $A \in M,\left(B \otimes E_{j i}\right) A \in M$. The $j$ th row of $\left(B \otimes E_{j i}\right) A$ is $\left(B A_{i 1}, \ldots, B A_{i n}\right)$. It follows that $\mathfrak{B} M_{i} \subseteq M_{j}$. Suppose there is some $A \in M_{i} \backslash M_{j}$. Then there is some $\psi \in \Lambda_{j}$ and $P \in \mathcal{M}(\mathfrak{B})$ such that $P \psi=\psi$ and $\psi(A) \neq 0$. However, if we choose a net $\left(B_{\lambda}\right) \subseteq \mathfrak{B}$ with $B_{\lambda} \rightarrow P$ in the ultraweak topology (which we can always do as $P \in \overline{\mathfrak{B}}^{w}$ ), then we see that

$$
\psi(A)=\psi(P A)=\lim _{\lambda} \psi\left(B_{\lambda} A\right)=0
$$

This proves that such an $A$ cannot exist and hence $M_{i} \subseteq M_{j}$. Swapping $i$ and $j$ in the previous analysis gives $M_{i}=M_{j}$. Once we set $\Lambda=\Lambda_{i}$ for some (and hence all) $i$, the inclusion

$$
M \subseteq \bigcap_{\psi \in \Lambda} \operatorname{ker} R_{\psi}
$$

follows immediately.

Given any $A \in \cap \operatorname{ker} R_{\psi}$ there must exist $A^{(1)}, \ldots, A^{(n)} \in M$ such that for each

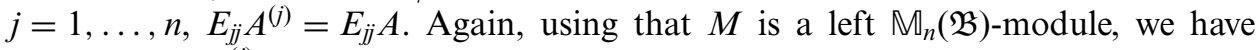
that $\left(B \otimes E_{i j}\right) A^{(j)} \in M$ for every $B \in \mathfrak{B}$. Since $M$ is ultraweakly closed, it follows that $P \otimes E_{\ddot{j}} A^{(j)} \in M$ for every $P \in \mathcal{M}(\mathfrak{B})$. As $\mathcal{M}(\mathfrak{B})$ is full, the sum

$$
\sum_{P \in \mathcal{M}(\mathfrak{B})} P \otimes E_{i j} A^{(j)}
$$

converges in the strong operator topology to $E_{i j} A^{(j)}$, and since all the partial sums are bounded, it also converges in the ultraweak topology. So $E_{i j} A^{(j)} \in M$. Writing

$$
A=\sum_{j=1}^{n} E_{j j} A^{(j)},
$$

we see that

$$
M=\bigcap_{\psi \in \Lambda} \operatorname{ker} R_{\psi} .
$$

The converse is straightforward. Fix $\psi \in \mathfrak{A}^{* n}$ with $P \psi=\psi$ for some $P \in \mathcal{M}(\mathfrak{B})$. If $A \in \operatorname{ker} R_{\psi}$ and $B \in \mathbb{M}_{n}(\mathfrak{B})$ then there is a matrix $\lambda=\left(\lambda_{i j}\right) \in \mathbb{M}_{n}$ such that

$$
B A \psi=\lambda A \psi=0 .
$$


It follows that for any antisymmetrically supported set $\Lambda \subseteq \mathfrak{A}^{* n}$,

$$
\bigcap_{\psi \in \Lambda} \operatorname{ker} R_{\psi}
$$

is a left $\mathbb{M}_{n}(\mathfrak{B})$-module.

3. The case where $\mathfrak{B} \subseteq Z(\mathfrak{A})$. In the following we fix two unital $C^{*}$-algebras $\mathfrak{A}$ and $\mathfrak{B}$, as before with $\mathfrak{B} \subseteq \mathfrak{A}$, but here we insist that $\mathfrak{B} \subseteq Z(\mathfrak{A})$ and that $\mathfrak{B}$ contains the identity in $\mathfrak{A}$. As usual, $\widehat{\mathfrak{A}}$ will denote the spectrum of $\mathfrak{A}$ (i.e. the set of equivalence classes of irreducible representations of $\mathfrak{A}$ ) equipped with the usual topology. Let $\Psi$ be the reduced atomic representation of $\mathfrak{A}$. That is,

$$
\Psi=\bigoplus_{[\pi] \in \widehat{\mathfrak{A}}} \pi,
$$

where each $\pi: \mathfrak{A} \rightarrow \mathcal{H}_{\pi}$ is a representative of the equivalence class $[\pi] \in \widehat{\mathfrak{A}}$. From now on we work only with this set of representatives and make no reference to the equivalence classes. We will show that this representation ensures that $\mathfrak{B}$ has a full set of antisymmetric projections and each $P \in \mathcal{M}(\mathfrak{B})$ has a particularly simple form.

For $\pi \in \widehat{\mathfrak{A}}$, let $E_{\pi}$ denote the projection in $\Psi(\mathfrak{A})^{\prime}$ defined by setting

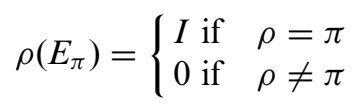

for every $\rho \in \widehat{\mathfrak{A}}$. Since $\mathfrak{B}$ is contained in the centre of $\mathfrak{A}$, we have that for any irreducible representation $\pi$ of $\mathfrak{A}, \pi(\mathfrak{B})=\mathbb{C} I$. Since every irreducible representation of $\mathfrak{B}$ extends to an irreducible representation of $\mathfrak{A}$ (on a necessarily larger Hilbert space), we see that the map $\pi \mapsto \pi_{\mathfrak{B}}$, where $\pi_{\mathfrak{B}}(A)=(\pi(A) \xi \mid \xi)$ for any unit vector $\xi \in \mathcal{H}_{\pi}$, defines a continuous surjection of $\widehat{\mathfrak{A}}$ onto $\Delta(\mathfrak{B})$. Following the ideas of Izuchi and Suárez [5] we will define for each $\varphi \in \Delta(\mathfrak{B})$, the fibre above $\varphi$ to be the set

$$
\mathcal{F}_{\varphi}=\left\{\pi \in \widehat{\mathfrak{A}}: \pi_{\mathfrak{B}}=\varphi\right\} .
$$

Set

$$
P_{\varphi}=\sum_{\pi \in \mathcal{F}_{\varphi}} E_{\pi}
$$

with the sum converging in the strong operator topology. Since for each $B \in \mathfrak{B}$ there is some $\lambda \in \mathbb{C}$ with $\pi(B)=\lambda I$ for every $\pi \in \mathcal{F}_{\varphi}$, it is clear that the projection $P_{\varphi}$ is $\mathfrak{B}$-antisymmetric. The fact that

$$
\sum_{\varphi \in \Delta(\mathfrak{B})} P_{\varphi}=I
$$

follows from the surjectivity of the map $\pi \mapsto \pi_{\mathfrak{B}}$. So to show that each $P_{\varphi}$ is maximal it is only necessary to show that for any two distinct characters $\varphi, \chi \in \Delta(\mathfrak{B})$ there exists some $B \in \mathfrak{B}$ and distinct complex numbers $\lambda_{1}$ and $\lambda_{2}$ such that $\Psi(B) P_{\varphi}=\lambda_{1} P_{\varphi}$ and $\Psi(B) P_{\chi}=\lambda_{2} P_{\chi}$. This follows easily since by the Gelfand representation there 
must exist $B \in \mathfrak{B}$ with $\varphi(B)=1$ and $\chi(B)=0$, and so $\Psi(B) P_{\varphi}=\varphi(B) P_{\varphi}=P_{\varphi}$ and $\Psi(B) P_{\chi}=\chi(B) P_{\chi}=0$.

We are still not in a position to apply Theorem 2 because we have not shown that each $\psi \in \mathfrak{A}^{*}$ is ultraweakly continuous on $\Psi(\mathfrak{A})$, and indeed this is in general not the case. Despite this, we will show, using the idea of Glicksberg in [4] for the commutative case, that the conclusions of Lemma 3 still hold. Before doing this, however, we must start by fixing some terminology. If $\psi \in \mathfrak{A}^{*}$, the null space of $\psi$ is the ideal $\mathcal{N}(\psi) \subseteq \mathfrak{A}$ consisting of all $A \in \mathfrak{A}$ such that $\psi(B A C)=0$ for every $B, C \in \mathfrak{A}$ and the support of $\psi$ is the projection

$$
S_{\psi}=\sum_{\mathcal{N}(\psi) \subseteq \operatorname{ker} \pi} E_{\pi} .
$$

So a functional $\psi \in \mathfrak{A}^{*}$ is antisymmetrically supported if and only if $S_{\psi} \leq P_{\varphi}$ for some $\varphi \in \Delta(\mathfrak{B})$. Since for every $A \in \mathfrak{A}$ and $\pi \in \widehat{\mathfrak{A}}$ with $\mathcal{N}(\psi) \subseteq \operatorname{ker} \pi, \pi\left(\left(I-S_{\psi}\right) A\right)=0$, it follows from [1, Proposition 2.11.2] that the norm of $\left(I-S_{\psi}\right) A$ in $\mathfrak{A} / \mathcal{N}(\psi)$ is 0 and so $S_{\psi} \psi=\psi$.

Lemma 8. Assume $\mathfrak{B} \subseteq Z(\mathfrak{A})$ and let $M \subseteq \mathfrak{A}$ be a closed left $\mathfrak{B}$-module. If $A \in \mathfrak{A}$ and $P_{\varphi} A \in P_{\varphi} M$ for every $\varphi \in \Delta(\mathfrak{B})$ then $A \in M$.

Proof. Let $\psi \in\left(\mathfrak{A}^{*}\right)_{1}$ be an extreme point of $\left(M^{\perp}\right)_{1}$. We will show that $S_{\psi}$ is a $\mathfrak{B}$-antisymmetric projection.

Let us first note that a projection $P \in \Psi(\mathfrak{A})^{\prime}$ is $\mathfrak{B}$-antisymmetric if and only if the ideal $\mathfrak{B} \cap(I-P) \mathfrak{B}$ is maximal in $\mathfrak{B}$. This is because if $P$ is $\mathfrak{B}$-antisymmetric then for each $B \in \mathfrak{B}$ there is some $\lambda(B) \in \mathbb{C}$ such that $P \Psi(B)=\lambda(B) P$, so the map $B \mapsto \lambda(B)$ is character on $\mathfrak{B}$ with kernel $\mathfrak{B} \cap(I-P) \mathfrak{B}$. Conversely, if $\mathfrak{B} \cap(I-P) \mathfrak{B}$ is maximal, then $P \mathfrak{B} \simeq \mathfrak{B} /(\mathfrak{B} \cap(I-P) \mathfrak{B}) \simeq \mathbb{C}$ and so $P \mathfrak{B}=\mathbb{C} P$.

Let $\tau: \mathfrak{B} \rightarrow \mathfrak{B} /\left(\mathfrak{B} \cap\left(I-S_{\psi}\right) \mathfrak{B}\right)$ be the quotient map. Choose a positive element $B \in(\mathfrak{B})_{1}$ such that $\tau(B) \neq 0$ and $\tau(B)$ is not invertible. Then by [1, Proposition 2.11.2] and the Gelfand-Naimark theorem, there must exist some $\pi \in \widehat{\mathfrak{A}}$ with $\mathcal{N}(\psi) \subseteq \operatorname{ker} \pi$ and $\pi(B)=0$. This implies that $\psi$ and $B \psi$ are linearly independent, otherwise there would be some non-zero $\lambda \in \mathbb{C}$ with $(B-\lambda I) \psi=0$, and since $B \in Z(\mathfrak{A}), B-\lambda I \in$ $\mathcal{N}(\psi) \subseteq \operatorname{ker} \pi$. We also have for any $A, C \in(\mathfrak{A})_{1}$,

$$
\begin{aligned}
|B \psi(A)+(I-B) \psi(C)| & =|\psi(B A+(I-B) C)| \\
& \leq\left\|S_{\psi}(B A+(I-B) C)\right\| \\
& =\sup _{E_{\pi} \leq S_{\psi}}\|\pi(B A+(I-B) C)\| \\
& \leq \sup _{E_{\pi} \leq S_{\psi}}\left(\pi_{\mathfrak{B}}(B)\|A\|+\left(1-\pi_{\mathfrak{B}}(B)\right)\|C\|\right) \leq 1 .
\end{aligned}
$$

Consequently, we have $1=\|\psi\| \leq\|B \psi\|+\|(I-B) \psi\| \leq 1$. Writing

$$
\psi=\|B \psi\|\left(\frac{B \psi}{\|B \psi\|}\right)+\|(I-B) \psi\|\left(\frac{(I-B) \psi}{\|(I-B) \psi\|}\right)
$$

we have expressed $\psi$ as a nontrivial convex sum of elements in $\mathfrak{A}^{*} \cap \mathcal{M}^{\perp}$, which is a contradiction. We conclude that every non-zero positive element of $\mathfrak{B} /(\mathfrak{B} \cap(I-$ $\left.\left.S_{\psi}\right) \mathfrak{B}\right)$ is invertible. It follows from the Gelfand-Mazur theorem that $\mathfrak{B} /(\mathfrak{B} \cap(I-$ $\left.S_{\psi}\right) \mathfrak{B}$ ) has co-dimension 1, which completes the proof. 
From this we can state versions of Theorems 2 and 7 for this setting.

THEOREM 9. If $\mathfrak{B} \subseteq Z(\mathfrak{A})$ then $M \subseteq \mathfrak{A}$ is a closed left $\mathfrak{B}$-module if and only if there exists an antisymmetrically supported set $\Lambda \subseteq \mathfrak{A}^{*}$ such that

$$
M=\bigcap_{\psi \in \Lambda} \operatorname{ker} \psi
$$

THEOREM 10. If $\mathfrak{B} \subseteq Z(\mathfrak{A})$ then $M \subseteq \mathbb{M}_{n}(\mathfrak{A})$ is a closed left $\mathbb{M}_{n}(\mathfrak{B})$-module if and only if there is an antisymmetrically supported set $\Lambda \subseteq \mathfrak{A}^{* n}$ such that

$$
M=\bigcap_{\psi \in \Lambda} \operatorname{ker} R_{\psi}
$$

The proofs of Theorems 9 and 10 are almost identical to those of Theorems 2 and 7 and so we omit them. There is however one difference that should be pointed out: The appeal to the ultraweak continuity of bounded linear functionals in the proof of Theorem 7 is not necessary for Theorem 10 because $\mathfrak{B}$ contains the identity.

ACKNOWLEDGEMENTS. The author would like to gratefully acknowledge the financial support of the UK Engineering and Physical Sciences Research Council (EPSRC), and the school of mathematics at the University of Leeds. He also wishes to thank Jonathan Partington for a great deal of guidance and support, and Matthew Daws for many useful discussions.

\section{REFERENCES}

1. J. Dixmier, $C^{*}$-algebras (North-Holland, New York, NY, 1969).

2. T. W. Gamelin, Uniform algebras (Prentice-Hall, Upper Saddle River, NJ, 1969).

3. J. B. Garnett, Bounded analytic functions (Academic Press, London, 1981).

4. I. Glicksberg, Measures orthogonal to algebras and sets of antisymmetry, Trans. Amer. Math. Soc. 105(3) (1962), 415-435.

5. K. Izuchi and D. Suárez, Norm closed invariant subspaces in $L^{\infty}$ and $H^{\infty}$, Glasgow Math. J. 46 (2004), 399-404.

6. R. V. Kadison and J. R. Ringrose, Fundamentals of the theory of operator algebras, vol. 2 (Academic Press, London, 1986).

7. J. R. Partington, Linear operators and linear systems: an analytical approach to control theory (Cambridge University Press, Cambridge, UK, 2004). 274.

8. W. Szymanski, Antisymmetric operator algebras 1, Ann. Polon. Math. 37 (1980), 263 311.

9. W. Szymanski, Antisymmetric operator algebras 2, Ann. Polon. Math. 37 (1980), 299- 